\title{
Interactive comment on "Contrasting transit times of water from peatlands and eucalypt forests in the Australian Alps determined by tritium: implications for vulnerability and the source of water in upland catchments" by I. Cartwright and U. Morgenstern
}

Anonymous Referee \#2

Received and published: 5 September 2016

The paper presents a comparison between mean transit time, MTT, between peatlands and eucalyptus forest in the Australian Alps. The authors used tritium as a tracer to model MTT using a lumped parameter approach. The authors also integrate geochemistry data and water stable isotopes in the analysis to yield important interpretations related to water storage and availability in these ecosystems. The paper is relevant to the scientific community because it provides information about underrepresented ecosystems with scare hydrologic data. The paper could eventually make an impor- 
tant contribution to the scientific literature. However, I believe there are issues with the paper structure and writing style. Thus I recommend major revisions before it can be considered for publication. I provide below some specific comments and suggestions:

Abstract: Lines 1-2: the first sentence sounds redundant (use of word "that"). Please rewrite.

Line 8: define the acronym before using it (TU).

Interactive

comment

1. Introduction:

I believe the introduction should provide more information about the use of geochemical data the context of mean transit time modelling.

Page 3, line 21-23: This sentence should be written.

Page 4, section 1.1: I think the authors should include a paragraph about the recent advances and challenges in the determination of transit times and the use of lumped parameter models. For instance, the authors should clarify that the calculated times are most likely representative of base flow conditions and spell out the underlying assumptions in the use of these models. There is a vast new literature dealing with time variant modelling of transit times.

Page 4, line 19-22: there is a missing "the" before "use". In addition are there any relevant references to this statement?

Page 4 line 19: consider using "to determine" instead of "to determining"

Page 6: I suggest you eliminate section 1.3 and have the objectives of the study be the last paragraph of the introduction.

Page 6 line 11-17: this information should appear before the paragraph with the objectives. Could be part of the last paragraph in page 5 .

Printer-friendly version

2. Setting:

Discussion paper 
I suggest this section be part of Methods.

Page 6 line 19-22. I suggest you rewrite this sentence.

Page 7 line 1-2, line 13-14: Please use the correct notation for the scientific name of species.

Interactive

3. Methods

comment

Line 9: Explain "aggregated" over what time frame? In addition, how many, when, how frequent were the samples collected?

Line 9-10: That just means grab samples, right?

Page 9 section 3.2. More information is required about the modeling procedure, how were the best parameters identified, what objective function was used, how many possible parameter combinations were implemented, can you include dotty plots? How did you chose among the 3 different transit time functions (exponential, piston flow, and dispersion). Why did you chose these 3 and not others?

\section{Results}

Here is where the major structural issues arise: The results section does not present any of the MTT related findings. This is odd considering that this is precisely the main topic of the paper. Residence time results are mentioned in the abstract, discussion, and conclusions. The manuscript must include a residence time results section in which the modelling findings are presented.

Page 11 lines 2-5: This sentence is awkward. Please rewrite.

Page 11, line 8: Avoid starting the sentence with a delta symbol, instead say" Water stable isotopes ( $\delta 18 \mathrm{O}$ and $\delta 2 \mathrm{H})$.

Printer-friendly version

Page 11: section 4.2. Figure 3 should be cited sooner. In addition it is not clear when or how many samples were collected. 
Page 11 line 11-12: is this slope different from the GMWL. The MMWL in Fig. 3 is very step is that correct. In addition, the text says that the samples line to the left of this line which is not true.

Page 11 line 13: please provide the range if deuterium execs.

Page 11 line 22-25: This sentence is too long.

Page 12 line 5: the word "and" makes no sense.

Page 12 Lines 3-10: Did you test the data for normality to make sure a parametric test was appropriate?

Page 12 line 11-14: this sentence is too long.

\section{Discussion:}

Page 13 line 19-21: Please avoid single sentence paragraphs

Page 14 line 22: reword sentence.

Page 15: The MTT results should be move to the result section. Also the selection of the best model should be justified both physically and statistically. What objective function was use to qualify the goodness of fit?

Tables: in general the captions need to be more comprehensive. For instance, the caption for Table 1 should indicate if the isotopic values averages? If so what is the time period over which they were calculated, how many samples are included, and are there metrics of uncertainty.

\section{Figures}

Figure 1: Please use different markets to indicate the location of peatlands and eucalyptus forest.

Figure 2b. Please use probability scale in the $x$-axis. 
Fig. 3 not clear what the dash (-) line is. The error bars for the grab samples should be representative of the accuracy (from the analysis of duplicate samples). If the precipitation signature corresponds to weighted means, then the error should be weighed errors. Please add the number of samples $(n=x x)$ associated to each (peat, Eucalypts, rain)

Figure 4: Not sure what the equation and $\mathrm{R} 2=0.89$ mean versus the $\mathrm{R} 2=0.69$. The legend has a "series 3" and "linear (series 3)", automatically generated from excel, that are not identified.

Interactive comment on Hydrol. Earth Syst. Sci. Discuss., doi:10.5194/hess-2016-361, 2016. 